\title{
Berry phase and adiabaticity of a spin diffusing in a nonuniform magnetic field
}

\author{
S. A. van Langen ${ }^{*}$ and H. P. A. Knops \\ Instituut-Lorentz, Leiden University, P.O. Box 9506, 2300 RA Leiden, The Netherlands \\ J. C. J. Paasschens \\ Instituut-Lorentz, Leiden University, P.O. Box 9506, 2300 RA Leiden, The Netherlands \\ and Philips Research Laboratories, 5656 AA Eindhoven, The Netherlands \\ C. W. J. Beenakker \\ Instituut-Lorentz, Leiden University, P.O. Box 9506, 2300 RA Leiden, The Netherlands
}

(Received 13 March 1998)

\begin{abstract}
An electron spin moving adiabatically in a strong, spatially nonuniform magnetic field accumulates a geometric phase or Berry phase, which might be observable as a conductance oscillation in a mesoscopic ring. Two contradicting theories exist for how strong the magnetic field should be to ensure adiabaticity if the motion is diffusive. To resolve this controversy, we study the effect of a nonuniform magnetic field on the spin polarization and on the weak-localization effect. The diffusion equation for the Cooperon is solved exactly. Adiabaticity requires that the spin-precession time is short compared to the elastic scattering time-it is not sufficient that it is short compared to the diffusion time around the ring. This strong condition severely complicates the experimental observation. [S0163-1829(99)10103-6]
\end{abstract}

\section{INTRODUCTION}

The adiabatic theorem of quantum mechanics implies that the final state of a particle that moves slowly along a closed path is identical to the initial eigenstate- up to a phase factor. The Berry phase is a time-independent contribution to this phase, depending only on the geometry of the path. ${ }^{1} \mathrm{~A}$ simple example is a spin-1/2 in a rotating magnetic field $\mathbf{B}$, where the Berry phase equals half the solid angle swept by B. It was proposed to measure the Berry phase in the conductance $G$ of a mesoscopic ring in a spatially rotating magnetic field. ${ }^{2,3}$ Oscillations of $G$ as a function of the swept solid angle were predicted, similar to the Aharonov-Bohm oscillations as a function of the enclosed flux. ${ }^{4}$

An important practical difference between the two effects is that the Aharonov-Bohm oscillations exist at arbitrarily small magnetic fields, whereas for the oscillations due to the Berry phase the magnetic field should be sufficiently strong to allow the spin to adiabatically follow the changing direction. Generally speaking, adiabaticity requires that the precession frequency $\omega_{\mathrm{B}}$ is large compared to the reciprocal of the characteristic time scale $t_{\mathrm{c}}$ on which $\mathbf{B}$ changes direction. We know that $\omega_{\mathrm{B}}=g \mu_{\mathrm{B}} B / 2 \hbar$, with $g$ the Landé factor and $\mu_{\mathrm{B}}$ the Bohr magneton. The question is, what is $t_{\mathrm{c}}$ ? In a ballistic ring there is only one candidate, the circumference $L$ of the ring divided by the Fermi velocity $v$. (For simplicity we assume that $L$ is also the scale on which the field direction changes.) In a diffusive ring there are two candidates: the elastic scattering time $\tau$ and the diffusion time $\tau_{\mathrm{d}}$ around the ring. They differ by a factor $\tau_{\mathrm{d}} / \tau \simeq(L / \ell)^{2}$, where $\ell$ $=v \tau$ is the mean free path. Since, by definition, $L \gg \ell$ in a diffusive system, the two time scales are far apart. Which of the two time scales is the relevant one is still under debate. ${ }^{5}$

Stern's original proposal ${ }^{3}$ was that

$$
\omega_{\mathrm{B}} \gg \frac{1}{\tau}
$$

is necessary to observe the Berry-phase oscillations. For realistic values of $g$ this requires magnetic fields in the quantum Hall regime, outside the range of validity of the semiclassical theory. We call Eq. (1.1) the "pessimistic criterion." In a later work, ${ }^{6}$ Loss, Schoeller, and Goldbart (LSG) concluded that adiabaticity is reached already at much weaker magnetic fields, when

$$
\omega_{\mathrm{B}} \gg \frac{1}{\tau_{\mathrm{d}}} \simeq \frac{1}{\tau}\left(\frac{\ell}{L}\right)^{2} .
$$

This "optimistic criterion" has motivated experimentalists to search for the Berry-phase oscillations in disordered conductors, ${ }^{7}$ and was invoked in a recent study of the conductivity of mesoscopic ferromagnets. ${ }^{8}$ In this paper, we reexamine the semiclassical theory of LSG to resolve the controversy.

The Berry-phase oscillations in the conductance result from a periodic modulation of the weak-localization correction, and require the solution of a diffusion equation for the Cooperon propagator. To solve this problem we need to consider the coupled dynamics of four spin degrees of freedom. (The Cooperon has four spin indices.) To gain insight we first examine in Sec. II the simpler problem of the dynamics of a single spin variable, by studying the randomization of a spin-polarized electron gas by a nonuniform magnetic field. We start at the level of the Boltzmann equation and then make the diffusion approximation. We show how the diffusion equation can be solved exactly for the first two moments of the polarization. The same procedure is used in Sec. III to arrive at a diffusion equation for the Cooperon. This equation coincides with the equation derived by LSG in the 


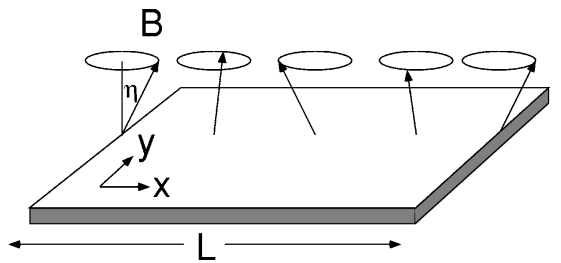

FIG. 1. Schematic drawing of a two-dimensional electron gas in the spatially rotating magnetic field of Eq. (2.1), with $f=1$.

weak-field regime $\omega_{\mathrm{B}} \tau \ll 1$, but is different in the strong-field regime $\omega_{\mathrm{B}} \tau \gtrsim 1$. We present an exact solution for the weaklocalization correction and compare with the findings of LSG.

Our conclusion both for the polarization and for the weaklocalization correction is that adiabaticity requires $\omega_{\mathrm{B}} \tau \gg 1$. Regrettably, the pessimistic criterion (1.1) is correct, in agreement with Stern's original conclusion. The optimistic criterion (1.2) advocated by LSG turns out to be the criterion for maximal randomization of the spin by the magnetic field, and not the criterion for adiabaticity.

\section{SPIN-RESOLVED TRANSMISSION}

\section{A. Formulation of the problem}

Consider a conductor in a magnetic field $\mathbf{B}$, containing a disordered segment (length $L$, mean free path $\ell$ at Fermi velocity $v$ ) in which the magnetic field changes its direction. An electron at the Fermi level with spin up (relative to the local magnetic field) is injected at one end and reaches the other end. What is the probability that its spin is up?

For simplicity we take for the conductor a twodimensional electron gas (in the $x-y$ plane, with the disordered region between $x=0$ and $x=L$ ), and we ignore the curvature of the electron trajectories by the Lorentz force. The problem becomes effectively one-dimensional by assuming that $\mathbf{B}$ depends on $x$ only. We choose a rotation of $\mathbf{B}$ in the $x-y$ plane, according to

$$
\begin{aligned}
\mathbf{B}(x, y, z & =0) \\
& =\left(B \sin \eta \cos \frac{2 \pi f x}{L}, B \sin \eta \sin \frac{2 \pi f x}{L}, B \cos \eta\right),
\end{aligned}
$$

with $\eta$ and $f$ arbitrary parameters. The geometry is sketched in Fig. 1. We treat the orbital motion semiclassically, within the framework of the Boltzmann equation. (This is justified if the Fermi wavelength is much smaller than $\ell$.) The spin dynamics requires a fully quantum mechanical treatment. We assume that the Zeeman energy $g \mu_{\mathrm{B}} B$ is much smaller than the Fermi energy $\frac{1}{2} m v^{2}$, so that the orbital motion is independent of the spin.

We introduce the probability density $P(x, \phi, \xi, t)$ for the electron to be at time $t$ at position $x$ with velocity $\mathbf{v}$ $=(v \cos \phi, v \sin \phi, 0)$, in the spin state with spinor $\xi$ $=\left(\xi_{1}, \xi_{2}\right)$. The dynamics of $\xi$ depends on the local magnetic field according to

$$
\frac{d \xi}{d t}=\frac{i g \mu_{\mathrm{B}}}{2 \hbar} \mathbf{B} \cdot \boldsymbol{\sigma} \xi
$$

where $\boldsymbol{\sigma}=\left(\sigma_{x}, \sigma_{y}, \sigma_{z}\right)$ is the vector of Pauli matrices. It is convenient to decompose $\xi=\chi_{1} \xi_{\uparrow}+\chi_{2} \xi_{\downarrow}$ into the local eigenstates $\xi_{\uparrow}, \xi_{\downarrow}$ of $\mathbf{B} \cdot \boldsymbol{\sigma}$,

$$
\begin{gathered}
\xi_{\uparrow}=\left(\begin{array}{c}
\cos \frac{\eta}{2} \mathrm{e}^{-i \pi f x / L} \\
\sin \frac{\eta}{2} \mathrm{e}^{i \pi f x / L}
\end{array}\right), \quad \xi_{\downarrow}=\left(\begin{array}{c}
-\sin \frac{\eta}{2} \mathrm{e}^{-i \pi f x / L} \\
\cos \frac{\eta}{2} \mathrm{e}^{i \pi f x / L}
\end{array}\right), \\
\mathbf{B} \cdot \boldsymbol{\sigma} \xi_{\uparrow}=B \xi_{\uparrow}, \quad \mathbf{B} \cdot \boldsymbol{\sigma} \xi_{\downarrow}=-B \xi_{\downarrow}
\end{gathered}
$$

and use the real and imaginary parts of the coefficients $\chi_{1}, \chi_{2}$ as variables in the Boltzmann equation. The dynamics of the vector of coefficients $c=\left(c_{1}, c_{2}, c_{3}, c_{4}\right)$ $=\left(\operatorname{Re} \chi_{1}, \operatorname{Im} \chi_{1}, \operatorname{Re} \chi_{2}, \operatorname{Im} \chi_{2}\right)$ is given by

$$
\frac{d c}{d t}=\frac{1}{\tau} M c, \quad M=M_{0}+M_{1} \cos \phi,
$$

$$
M_{0}=\omega_{\mathrm{B}} \tau\left(\begin{array}{cccc}
0 & -1 & 0 & 0 \\
1 & 0 & 0 & 0 \\
0 & 0 & 0 & 1 \\
0 & 0 & -1 & 0
\end{array}\right)
$$

$$
M_{1}=\frac{\pi f \ell}{L}\left(\begin{array}{cccc}
0 & -\cos \eta & 0 & \sin \eta \\
\cos \eta & 0 & -\sin \eta & 0 \\
0 & \sin \eta & 0 & \cos \eta \\
-\sin \eta & 0 & -\cos \eta & 0
\end{array}\right) \text {, }
$$

where $\omega_{\mathrm{B}}=g \mu_{\mathrm{B}} B / 2 \hbar$ is the precession frequency of the spin. The Boltzmann equation takes the form

$$
\begin{aligned}
\tau \frac{\partial}{\partial t} P(x, \phi, c, t)= & -\ell \cos \phi \frac{\partial P}{\partial x}-\sum_{i, j} \frac{\partial}{\partial c_{i}}\left(M_{i j} c_{j} P\right) \\
& -P+\int_{0}^{2 \pi} \frac{d \phi^{\prime}}{2 \pi} P\left(x, \phi^{\prime}, c, t\right),
\end{aligned}
$$

where we have assumed isotropic scattering (rate $1 / \tau$ $=v / \ell$ ).

We look for a stationary solution to the Boltzmann equation, so the left-hand side of Eq. (2.5) is zero and we omit the argument $t$ of $P$. A stationary flux of particles with an isotropic velocity distribution is injected at $x=0$, their spins all aligned with the local magnetic field (so $\chi_{2}=0$ at $x=0$ ). Without loss of generality we may assume that $\chi_{1}=1$ at $x$ $=0$. No particles are incident from the other end, at $x=L$. Thus the boundary conditions are

$P(x=0, \phi, c)=\delta\left(c_{1}-1\right) \delta\left(c_{2}\right) \delta\left(c_{3}\right) \delta\left(c_{4}\right) \quad$ if $\cos \phi>0$,

$P(x=L, \phi, c)=0 \quad$ if $\cos \phi<0$.

This completes the formulation of the problem. We compare two methods of solution. The first is an exact numerical solution of the Boltzmann equation using the Monte Carlo 
method. The second is an approximate analytical solution using the diffusion approximation, valid for $L \gg \ell$. We begin with the latter.

\section{B. Diffusion approximation}

The diffusion approximation amounts to the assumption that $P$ has a simple cosine-dependence on $\phi$,

$$
P(x, \phi, c)=N(x, c)+J(x, c) \cos \phi .
$$

To determine the density $N$ and current $J$ we substitute Eq. (2.7) into Eq. (2.5) and integrate over $\phi$. This gives

$$
\ell \frac{\partial J}{\partial x}=-\frac{\partial}{\partial c}\left(2 M_{0} c N+M_{1} c J\right) .
$$

Similarly, multiplication with $\cos \phi$ before integration gives

$$
\ell \frac{\partial N}{\partial x}=-\frac{\partial}{\partial c}\left(M_{0} c J+M_{1} c N\right)-J .
$$

Thus we have a closed set of partial differential equations for the unknown functions $N(x, c)$ and $J(x, c)$. Boundary conditions are obtained by multiplying Eq. (2.6) with $\cos \phi$ and integrating over $\phi$ :

$$
\begin{aligned}
& N(x=0, c)+\frac{\pi}{4} J(x=0, c) \\
& \quad=\delta\left(c_{1}-1\right) \delta\left(c_{2}\right) \delta\left(c_{3}\right) \delta\left(c_{4}\right), \\
& N(x=L, c)-\frac{\pi}{4} J(x=L, c)=0 .
\end{aligned}
$$

We seek the spin polarization $p=c_{1}^{2}+c_{2}^{2}-c_{3}^{2}-c_{4}^{2}$ of the transmitted electrons, characterized by the distribution

$$
P(p)=\frac{\int d c J(x=L, c) \delta\left(c_{1}^{2}+c_{2}^{2}-c_{3}^{2}-c_{4}^{2}-p\right)}{\int d c J(x=L, c)} .
$$

(The notation $\int d c \equiv \int d c_{1} \int d c_{2} \int d c_{3} \int d c_{4}$ indicates an integration over the spin variables.) We compute the first two moments of $P(p)$. The first moment $\bar{p}$ is the fraction of transmitted electrons with spin up minus the fraction with spin down, averaged quantum mechanically over the spin state and statistically over the disorder. The variance $\operatorname{Var} p$ $=\overline{p^{2}}-\bar{p}^{2}$ gives an indication of the magnitude of the statistical fluctuations.

Integration of Eqs. (2.8)-(2.10) over the spin variables yields the equations and boundary conditions for the functions $N(x)=\int d c N(x, c)$ and $J(x)=\int d c J(x, c)$ :

$$
\begin{gathered}
\ell \frac{d N}{d x}=-J, \quad \frac{d J}{d x}=0, \\
N(0)+\frac{\pi}{4} J(0)=1, \quad N(L)-\frac{\pi}{4} J(L)=0 .
\end{gathered}
$$

The solution

$$
J(x)=\left(\frac{\pi}{2}+\frac{L}{\ell}\right)^{-1}
$$

determines the denominator of Eq. (2.11).

To determine $\bar{p}$ we multiply Eqs. (2.8) and (2.9) with $\chi_{\alpha} \chi_{\beta}^{*}$ and integrate over $c$ (recall that $\chi_{1}=c_{1}+i c_{2}, \chi_{2}=c_{3}$ $+i c_{4}$ ). It follows upon partial integration that

$$
\begin{aligned}
& \int d c \chi_{\alpha} \chi_{\beta}^{*} \frac{\partial}{\partial c}\left(M_{0} c f\right) \\
& =-\sum_{\rho, \sigma}\left(S_{\alpha \rho} \delta_{\beta \sigma}-\delta_{\alpha \rho} S_{\beta \sigma}\right) \int d c \chi_{\rho} \chi_{\sigma}^{*} f,
\end{aligned}
$$

$$
\begin{aligned}
& \int d c \chi_{\alpha} \chi_{\beta}^{*} \frac{\partial}{\partial c}\left(M_{1} c f\right) \\
& =-\sum_{\rho, \sigma}\left(T_{\alpha \rho} \delta_{\beta \sigma}-\delta_{\alpha \rho} T_{\beta \sigma}\right) \int d c \chi_{\rho} \chi_{\sigma}^{*} f,
\end{aligned}
$$

for arbitrary functions $f(x, c)$. The $2 \times 2$ matrices $S, T$ are defined by

$$
S=i \omega_{\mathrm{B}} \tau \sigma_{z}, \quad T=\frac{i \pi f \ell}{L}\left(\sigma_{z} \cos \eta-\sigma_{x} \sin \eta\right) .
$$

In this way we find that the moments

$$
\begin{gathered}
N_{\alpha \beta}(x)=\int d c \chi_{\alpha} \chi_{\beta}^{*} N(x, c), \\
J_{\alpha \beta}(x)=\int d c \chi_{\alpha} \chi_{\beta}^{*} J(x, c),
\end{gathered}
$$

satisfy the ordinary differential equations

$$
\begin{aligned}
& \ell \frac{d N_{\alpha \beta}}{d x}=\sum_{\rho, \sigma}\left(T_{\alpha \rho} \delta_{\beta \sigma}-\delta_{\alpha \rho} T_{\beta \sigma}\right) N_{\rho \sigma} \\
& +\sum_{\rho, \sigma}\left(S_{\alpha \rho} \delta_{\beta \sigma}-\delta_{\alpha \rho} S_{\beta \sigma}\right) J_{\rho \sigma}-J_{\alpha \beta}, \\
& \ell \frac{d J_{\alpha \beta}}{d x}=2 \sum_{\rho, \sigma}\left(S_{\alpha \rho} \delta_{\beta \sigma}-\delta_{\alpha \rho} S_{\beta \sigma}\right) N_{\rho \sigma} \\
& +\sum_{\rho, \sigma}\left(T_{\alpha \rho} \delta_{\beta \sigma}-\delta_{\alpha \rho} T_{\beta \sigma}\right) J_{\rho \sigma},
\end{aligned}
$$

with boundary conditions

$$
\begin{gathered}
N_{\alpha \beta}(x=0)+\frac{\pi}{4} J_{\alpha \beta}(x=0)=\delta_{\alpha 1} \delta_{\beta 1}, \\
N_{\alpha \beta}(x=L)-\frac{\pi}{4} J_{\alpha \beta}(x=L)=0 .
\end{gathered}
$$

The mean polarization $\bar{p}$ is determined by $J_{\alpha \beta}$ according to 


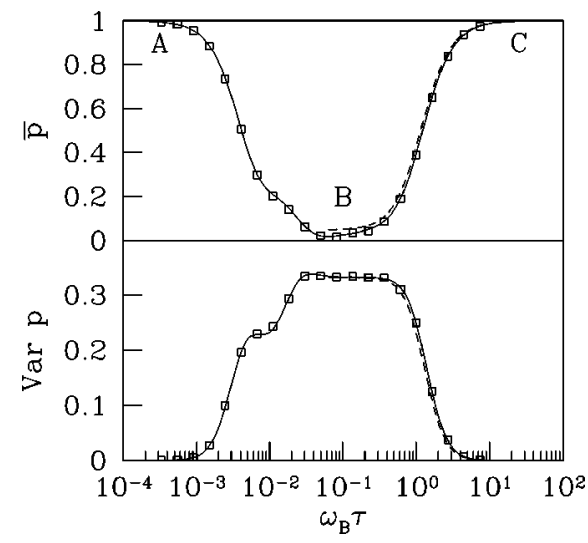

FIG. 2. Average and variance of the spin polarization $p$ of the current transmitted through a two-dimensional region of length $L$ $=25 \ell$, as a function of $\omega_{\mathrm{B}} \tau$, for a magnetic field given by Eq. (2.1) with $\eta=\pi / 3$ and $f=1$. The data points result from Monte Carlo simulations of the Boltzmann equation (2.5), the solid curves result from the diffusion approximation (2.7), and the dashed curves are the asymptotic formulas (2.20) and (2.27). Notice the transient regime $(A)$, the randomized regime $(B)$, and the adiabatic regime $(C)$.

$$
\begin{aligned}
\bar{p} & =\frac{J_{11}(L)-J_{22}(L)}{J(L)} \\
& =\left(\frac{\pi}{2}+\frac{L}{\ell}\right)\left[J_{11}(L)-J_{22}(L)\right] .
\end{aligned}
$$

Since Eq. (2.17) is linear in the eight functions $N_{\alpha \beta}(x), J_{\alpha \beta}(x)(\alpha, \beta=1,2)$, a solution requires the eigenvalues and right eigenvectors of the $8 \times 8$ matrix of coefficients. These can be readily computed numerically for any values of $L / \ell$ and $\omega_{\mathrm{B}} \tau$. We have found an analytic asymptotic solution for $L / \ell \gg 1$ and $\omega_{\mathrm{B}} \tau \gg(f \ell / L)^{2}$, given by

$$
\bar{p}=\frac{k}{\sinh k}, \quad k=\frac{2 \pi f \sin \eta}{\sqrt{1+\left(2 \omega_{\mathrm{B}} \tau\right)^{2}}} .
$$

In Fig. 2 we compare the numerical solution (solid curve) with Eq. (2.20) (dashed curve) for $L / \ell=25$ and $\eta=\pi / 3, f$ $=1$. The two curves are almost indistinguishable, except for the smallest values of $\omega_{\mathrm{B}} \tau$.

In a similar way, we compute the second moment of $P(p)$ by multiplying Eqs. (2.8) and (2.9) with $\chi_{\alpha} \chi_{\beta}^{*} \chi_{\gamma} \chi_{\delta}^{*}$ and integrating over $c$. The result is a closed set of equations,

$$
\frac{d}{d x} N_{\alpha \beta \gamma \delta}=\sum_{\mu, \nu, \rho, \sigma}\left(L_{\alpha \beta \gamma \delta}^{\mu \nu \rho \sigma} N_{\mu \nu \rho \sigma}+K_{\alpha \beta \gamma \delta}^{\mu \nu \rho \sigma} J_{\mu \nu \rho \sigma}\right)-J_{\alpha \beta \gamma \delta}
$$

$\ell \frac{d}{d x} J_{\alpha \beta \gamma \delta}=\sum_{\mu, \nu, \rho, \sigma}\left(2 K_{\alpha \beta \gamma \delta}^{\mu \nu \rho \sigma} N_{\mu \nu \rho \sigma}+L_{\alpha \beta \gamma \delta}^{\mu \nu \rho \sigma} J_{\mu \nu \rho \sigma}\right)$,

where we have defined

$$
\begin{aligned}
K_{\alpha \beta \gamma \delta}^{\mu \nu \rho \sigma}= & S_{\alpha \mu} \delta_{\beta \nu} \delta_{\gamma \rho} \delta_{\delta \sigma}-\delta_{\alpha \mu} S_{\beta \nu} \delta_{\gamma \rho} \delta_{\delta \sigma} \\
& +\delta_{\alpha \mu} \delta_{\beta \nu} S_{\gamma \rho} \delta_{\delta \sigma}-\delta_{\alpha \mu} \delta_{\beta \nu} \delta_{\gamma \rho} S_{\delta \sigma},
\end{aligned}
$$

$$
\begin{gathered}
L_{\alpha \beta \gamma \delta}^{\mu \nu \rho \sigma}=T_{\alpha \mu} \delta_{\beta \nu} \delta_{\gamma \rho} \delta_{\delta \sigma}-\delta_{\alpha \mu} T_{\beta \nu} \delta_{\gamma \rho} \delta_{\delta \sigma} \\
+\delta_{\alpha \mu} \delta_{\beta \nu} T_{\gamma \rho} \delta_{\delta \sigma}-\delta_{\alpha \mu} \delta_{\beta \nu} \delta_{\gamma \rho} T_{\delta \sigma}, \\
N_{\alpha \beta \gamma \delta}(x)=\int d c \chi_{\alpha} \chi_{\beta}^{*} \chi_{\gamma} \chi_{\delta}^{*} N(x, c), \\
J_{\alpha \beta \gamma \delta}(x)=\int d c \chi_{\alpha} \chi_{\beta}^{*} \chi_{\gamma} \chi_{\delta}^{*} J(x, c) .
\end{gathered}
$$

The boundary conditions on the functions $N_{\alpha \beta \gamma \delta}$ and $J_{\alpha \beta \gamma \delta}$ are

$N_{\alpha \beta \gamma \delta}(x=0)+\frac{\pi}{4} J_{\alpha \beta \gamma \delta}(x=0)=\delta_{\alpha 1} \delta_{\beta 1} \delta_{\gamma 1} \delta_{\delta 1}$,

$N_{\alpha \beta \gamma \delta}(x=L)-\frac{\pi}{4} J_{\alpha \beta \gamma \delta}(x=L)=0$.

The second moment $\overline{p^{2}}$ is determined by

$$
\begin{aligned}
\overline{p^{2}}= & \left(\frac{\pi}{2}+\frac{L}{\ell}\right)\left[J_{1111}(x=L)-J_{1122}(x=L)\right. \\
& \left.-J_{2211}(x=L)+J_{2222}(x=L)\right] .
\end{aligned}
$$

The numerical solution is plotted also in Fig. 2, together with the asymptotic expression

$$
\operatorname{Var} p=\frac{1}{3}+\frac{2 k \sqrt{3}}{3 \sinh (k \sqrt{3})}-\frac{k^{2}}{\sinh ^{2} k} .
$$

It is evident from Eqs. (2.20) and (2.27), and from Fig. 2, that the regime with $\bar{p}=1, \operatorname{Var} p=0$ is entered for $\omega_{\mathrm{B}} \tau \gtrsim f$ [for $\sin \eta=O(1)$ ], in agreement with Stern's criterion (1.1) for adiabaticity. For smaller $\omega_{\mathrm{B}} \tau$ adiabaticity is lost. There is a transient regime $\omega_{\mathrm{B}} \tau \ll(f \ell / L)^{2}$, in which the precession frequency is so low that the spin remains in the same state during the entire diffusion process. For $(f \ell / L)^{2} \ll \omega_{\mathrm{B}} \tau \ll f$ the average polarization reaches a plateau value close to zero with a finite variance. For a sufficiently nonuniform field, $f \sin \eta \gg 1$, we find in this regime $\bar{p}=0$ and $\operatorname{Var} p=1 / 3$, which means that the spin state is completely randomized. The transient regime, the randomized regime, and the adiabatic regime are indicated in Fig. 2 by the letters $A, B$, and $C$.

\section{Comparison with Monte Carlo simulations}

In order to check the diffusion approximation we solved the full Boltzmann equation by means of a Monte Carlo simulation. A particle is moved from $x=0$ over a distance $\ell_{1}$ in the direction $\phi_{1}$, then over a distance $\ell_{2}$ in the direction $\phi_{2}$, and so on, until it is reflected back to $x=0$ or transmitted to $x=L$. The step lengths $\ell_{i}$ are chosen randomly from a Poisson distribution with mean $\ell$. The directions $\phi_{i}$ are chosen uniformly from $[0,2 \pi]$, except for the initial direction $\phi_{1}$, which is distributed $\propto \cos \phi_{1}$. The spin components are given by

$$
\left(\begin{array}{l}
\chi_{1} \\
\chi_{2}
\end{array}\right)=\prod_{i} \mathrm{e}^{\left(S+T \cos \phi_{i}\right) \ell_{i} / \ell}\left(\begin{array}{l}
1 \\
0
\end{array}\right) .
$$


To find $\overline{p^{n}}$, one has to average $\left(\left|\chi_{1}\right|^{2}-\left|\chi_{2}\right|^{2}\right)^{n}$ over the transmitted particles. The results for $L / \ell=25$ are shown in Fig. 2 (data points). They agree very well with the results of the previous subsection, thus confirming the validity of the diffusion approximation for $L / \ell \gg 1$.

\section{WEAK LOCALIZATION}

\section{A. Formulation of the problem}

We turn to the effect of the nonuniform magnetic field on the weak-localization correction of a multiply connected system. We consider the same geometry as in Fig. 1, but now with periodic boundary conditions - to model a ring of circumference $L$. Only the effects of the magnetic field on the spin are included, to isolate the Berry phase from the conventional Aharonov-Bohm phase. As in the previous subsection, we assume that the orbital motion is independent of the spin dynamics. We follow LSG in applying the semiclassical theory of Chakravarty and Schmidt ${ }^{9}$ to the problem; however, we start at the level of the Boltzmann equation-rather than at the level of the diffusion equation-and make the diffusion approximation at a later stage of the calculation.

The weak-localization correction $\Delta G$ to the conductance is given by

$$
\Delta G=-\frac{e^{2} D}{\pi \hbar L} \int_{0}^{\infty} d t \mathrm{e}^{-t / \tau_{\varphi} C(t)}
$$

where $\tau_{\varphi}$ is the phase coherence time and the diffusion coefficient $D=v l / d$ in $d$ dimensions. (In our geometry $d=2$.) The "return quasiprobability" $C(t)$ is expressed as a sum over "Boltzmannian walks" $\mathbf{R}(t)$ with $\mathbf{R}(0)=\mathbf{R}(t)$,

$$
C(t)=\sum_{\{\mathbf{R}(t)\}} W \operatorname{Tr}\left(U^{+} U^{-}\right) .
$$

Here $W[\mathbf{R}(t)]$ is the weight of the Boltzmannian walk for a spinless particle. The $2 \times 2$ matrices $U^{ \pm}[\mathbf{R}(t)]$ are defined by

$$
U^{ \pm}=\mathcal{T} \exp \left\{ \pm \frac{i g \mu_{\mathrm{B}}}{2 \hbar} \int_{0}^{t} d t^{\prime} \mathbf{B}\left(\mathbf{R}\left(t^{\prime}\right)\right) \cdot \boldsymbol{\sigma}\right\}
$$

where $\mathcal{T}$ denotes a time ordering. The factor $\operatorname{Tr}\left(U^{+} U^{-}\right)$in Eq. (3.2) accounts for the phase difference of time-reversed paths.

The Cooperon can be written in terms of a propagator $\chi$,

$$
C(t)=\frac{1}{2 \pi} \int_{0}^{2 \pi} d \phi \int_{0}^{2 \pi} d \phi_{\mathrm{i}} \sum_{\alpha, \beta} \chi_{\alpha \beta \beta \alpha}\left(x_{\mathrm{i}}, x_{\mathrm{i}} ; \phi, \phi_{\mathrm{i}} ; t\right),
$$

that satisfies the kinetic equation

$$
\begin{aligned}
\left(\frac{\partial}{\partial t}\right. & +\mathcal{B}) \chi_{\alpha \beta \gamma \delta}\left(x, x_{\mathrm{i}} ; \phi, \phi_{\mathrm{i}} ; t\right)-\frac{i g \mu_{\mathrm{B}}}{2 \hbar} \\
& \times \sum_{\alpha^{\prime}, \gamma^{\prime}}\left[(\mathbf{B}(x) \cdot \boldsymbol{\sigma})_{\alpha \alpha^{\prime}} \delta_{\gamma \gamma^{\prime}}-\delta_{\alpha \alpha^{\prime}}(\mathbf{B}(x) \cdot \boldsymbol{\sigma})_{\gamma \gamma^{\prime}}\right] \chi_{\alpha^{\prime} \beta \gamma^{\prime} \delta} \\
& =\delta(t) \delta\left(x-x_{\mathrm{i}}\right) \delta\left(\phi-\phi_{\mathrm{i}}\right) \delta_{\alpha \beta} \delta_{\gamma \delta} .
\end{aligned}
$$

The Boltzmann operator $\mathcal{B}$ is given by

$$
\mathcal{B}=v \cos \phi \frac{\partial}{\partial x}+\frac{1}{\tau}-\frac{1}{\tau} \int_{0}^{2 \pi} \frac{d \phi}{2 \pi}
$$

The propagator $\chi$ is a moment of the probability distribution $P\left(x, \phi, U^{+}, U^{-}, t\right)$,

$$
\chi_{\alpha \beta \gamma \delta}=\int d U^{+} \int d U^{-} U_{\alpha \beta}^{+} U_{\gamma \delta}^{-} P
$$

that satisfies the Boltzmann equation

$$
\begin{gathered}
{\left[\frac{\partial}{\partial t}+\mathcal{B}+\frac{\partial}{\partial U^{+}}\left(\frac{d U^{+}}{d t}\right)+\frac{\partial}{\partial U^{-}}\left(\frac{d U^{-}}{d t}\right)\right]} \\
\times P\left(x, \phi, U^{+}, U^{-}, t\right)=0,
\end{gathered}
$$

with initial condition

$$
\begin{aligned}
& P\left(x, \phi, U^{+}, U^{-}, 0\right) \\
& \quad=\delta\left(x-x_{\mathrm{i}}\right) \delta\left(\phi-\phi_{\mathrm{i}}\right) \delta\left(U^{+}-1\right) \delta\left(U^{-}-1\right) .
\end{aligned}
$$

The notation $d U^{+}$or $d U^{-}$indicates the differential of the real and imaginary parts of the elements of the $2 \times 2$ matrix $U^{+}$or $U^{-}$. We will write this in a more explicit way in the next subsection.

The Boltzmann equation (3.8) has the same form as the one that we studied in Sec. II. The difference is that we have four times as many internal degrees of freedom. Instead of a single spinor $\xi$ we now have two spinor matrices $U^{+}$and $U^{-}$. A first doubling of the number of degrees of freedom occurs because we have to follow the evolution of both spin up and spin down. A second doubling occurs because we have to follow both the normal and the time-reversed evolution.

\section{B. Diffusion approximation}

We make the diffusion approximation to the Boltzmann equation (3.8), by following the steps outlined in Sec. II. The $4 \times 2$ matrix $u^{ \pm}$containing the real and imaginary parts of $U^{ \pm}$

$$
u^{ \pm}=\left(\begin{array}{ll}
\operatorname{Re} U_{11}^{ \pm} & \operatorname{Re} U_{12}^{ \pm} \\
\operatorname{Im} U_{11}^{ \pm} & \operatorname{Im} U_{12}^{ \pm} \\
\operatorname{Re} U_{21}^{ \pm} & \operatorname{Re} U_{22}^{ \pm} \\
\operatorname{Im} U_{21}^{ \pm} & \operatorname{Im} U_{22}^{ \pm}
\end{array}\right),
$$

has a time evolution governed by

$$
\tau \frac{d u^{ \pm}}{d t}= \pm Z(x) u^{ \pm}
$$




$$
Z(x)=\omega_{\mathrm{B}} \tau\left(\begin{array}{cccc}
0 & -\cos \eta & \sin \eta \sin \frac{2 \pi f x}{L} & -\sin \eta \cos \frac{2 \pi f x}{L} \\
\cos \eta & 0 & \sin \eta \cos \frac{2 \pi f x}{L} & \sin \eta \sin \frac{2 \pi f x}{L} \\
-\sin \eta \sin \frac{2 \pi f x}{L} & -\sin \eta \cos \frac{2 \pi f x}{L} & 0 & \cos \eta \\
\sin \eta \cos \frac{2 \pi f x}{L} & -\sin \eta \sin \frac{2 \pi f x}{L} & -\cos \eta & 0
\end{array}\right) .
$$

The Boltzmann equation (3.8) becomes, in a more explicit notation,

$$
\begin{aligned}
& \tau \frac{\partial}{\partial t} P\left(x, \phi, u^{+}, u^{-}, t\right) \\
& =-\ell \cos \phi \frac{\partial P}{\partial x}-\sum_{i, j, k} \frac{\partial}{\partial u_{i j}^{+}} Z_{i k}(x) u_{k j}^{+} P \\
& \quad+\sum_{i, j, k} \frac{\partial}{\partial u_{i j}^{-}} Z_{i k}(x) u_{k j}^{-} P-P \\
& \quad+\int_{0}^{2 \pi} \frac{d \phi^{\prime}}{2 \pi} P\left(x, \phi^{\prime}, u^{+}, u^{-}, t\right) .
\end{aligned}
$$

We now make the diffusion ansatz in the form

$$
\int_{0}^{\infty} d t \mathrm{e}^{-t / \tau_{\varphi}} \int_{0}^{2 \pi} d \phi_{\mathrm{i}} P=N+J \cos \phi .
$$

By integrating the Boltzmann equation over $\phi$, once with weight 1 and once with weight $\cos \phi$, we obtain two coupled equations for the functions $N\left(x, u^{+}, u^{-}\right)$and $J\left(x, u^{+}, u^{-}\right)$. Next we multiply both equations with $U_{\alpha \beta}^{+} U_{\gamma \delta}^{-}$and integrate over the real and imaginary parts of the matrix elements. The moments $N_{\alpha \beta \gamma \delta}$ and $J_{\alpha \beta \gamma \delta}$ defined by

$$
\begin{aligned}
& N_{\alpha \beta \gamma \delta}(x)=\int d U^{+} \int d U^{-} U_{\alpha \beta}^{+} U_{\gamma \delta}^{-} N, \\
& J_{\alpha \beta \gamma \delta}(x)=\int d U^{+} \int d U^{-} U_{\alpha \beta}^{+} U_{\gamma \delta}^{-} J,
\end{aligned}
$$

are found to obey the ordinary differential equations

$$
\begin{aligned}
\ell \frac{d N_{\alpha \beta \gamma \delta}}{d x}= & \frac{i g \mu_{\mathrm{B}} \tau}{2 \hbar} \sum_{\alpha^{\prime}, \gamma^{\prime}}\left[(\mathbf{B}(x) \cdot \boldsymbol{\sigma})_{\alpha \alpha^{\prime}} \delta_{\gamma \gamma^{\prime}}\right. \\
& \left.-\delta_{\alpha \alpha^{\prime}}(\mathbf{B}(x) \cdot \boldsymbol{\sigma})_{\gamma \gamma^{\prime}}\right] J_{\alpha^{\prime} \beta \gamma^{\prime} \delta}-\left(1+\tau / \tau_{\varphi}\right) J_{\alpha \beta \gamma \delta}
\end{aligned}
$$

$$
\begin{aligned}
\ell \frac{d J_{\alpha \beta \gamma \delta}}{d x}= & \frac{i g \mu_{\mathrm{B}} \tau}{\hbar} \sum_{\alpha^{\prime}, \gamma^{\prime}}\left[(\mathbf{B}(x) \cdot \boldsymbol{\sigma})_{\alpha \alpha^{\prime}} \delta_{\gamma \gamma^{\prime}}\right. \\
& \left.-\delta_{\alpha \alpha^{\prime}}(\mathbf{B}(x) \cdot \boldsymbol{\sigma})_{\gamma \gamma^{\prime}}\right] N_{\alpha^{\prime} \beta \gamma^{\prime} \delta} \\
& -\left(2 \tau / \tau_{\varphi}\right) N_{\alpha \beta \gamma \delta}+2 \tau \delta_{\alpha \beta} \delta_{\gamma \delta} \delta\left(x-x_{\mathrm{i}}\right)
\end{aligned}
$$

The periodic boundary conditions are

$$
N_{\alpha \beta \gamma \delta}(0)=N_{\alpha \beta \gamma \delta}(L), \quad J_{\alpha \beta \gamma \delta}(0)=J_{\alpha \beta \gamma \delta}(L) .
$$

The Cooperon $C$ and the propagator $\chi$ of Eqs. (3.4) and (3.7) are related to the density $N$ by

$$
\begin{array}{r}
N_{\alpha \beta \gamma \delta}(x)=\int_{0}^{\infty} d t c^{-t / \tau_{\varphi}} \frac{1}{2 \pi} \int_{0}^{2 \pi} d \phi \\
\quad \times \int_{0}^{2 \pi} d \phi_{\mathrm{i}} \chi_{\alpha \beta \gamma \delta}\left(x, x_{\mathrm{i}} ; \phi, \phi_{\mathrm{i}} ; t\right), \\
\sum_{\alpha, \beta} N_{\alpha \beta \beta \alpha}\left(x_{\mathrm{i}}\right)=\int_{0}^{\infty} d t e^{-t / \tau_{\varphi}} C(t) .
\end{array}
$$

Hence the weak-localization correction (3.1) is obtained from $N$ by

$$
\Delta G=-\frac{e^{2} D}{\pi \hbar L} \sum_{\alpha, \beta} N_{\alpha \beta \beta \alpha}\left(x_{\mathrm{i}}\right) .
$$

The transformation to the local basis of spin states (2.3) takes the form of a unitary transformation of the moments $N$ and $J$,

$$
\begin{gathered}
\tilde{N}_{\alpha \beta \gamma \delta}=\sum_{\alpha^{\prime}, \beta^{\prime}, \gamma^{\prime}, \delta^{\prime}} Q_{\alpha \alpha^{\prime}} Q_{\gamma \gamma^{\prime}} N_{\alpha^{\prime} \beta^{\prime} \gamma^{\prime} \delta^{\prime}} Q_{\beta^{\prime} \beta}^{\dagger} Q_{\delta^{\prime} \delta}^{\dagger}, \\
\widetilde{J}_{\alpha \beta \gamma \delta}=\sum_{\alpha^{\prime}, \beta^{\prime}, \gamma^{\prime}, \delta^{\prime}} Q_{\alpha \alpha^{\prime}} Q_{\gamma \gamma^{\prime}} J_{\alpha^{\prime} \beta^{\prime} \gamma^{\prime} \delta^{\prime}} Q_{\beta^{\prime} \beta}^{\dagger} Q_{\delta^{\prime} \delta}^{\dagger},
\end{gathered}
$$

$$
Q(x)=\left(\begin{array}{cc}
e^{i \pi f x / L} \cos \frac{\eta}{2} & e^{-i \pi f x / L} \sin \frac{\eta}{2} \\
-e^{i \pi f x / L} \sin \frac{\eta}{2} & e^{-i \pi f x / L} \cos \frac{\eta}{2}
\end{array}\right) .
$$



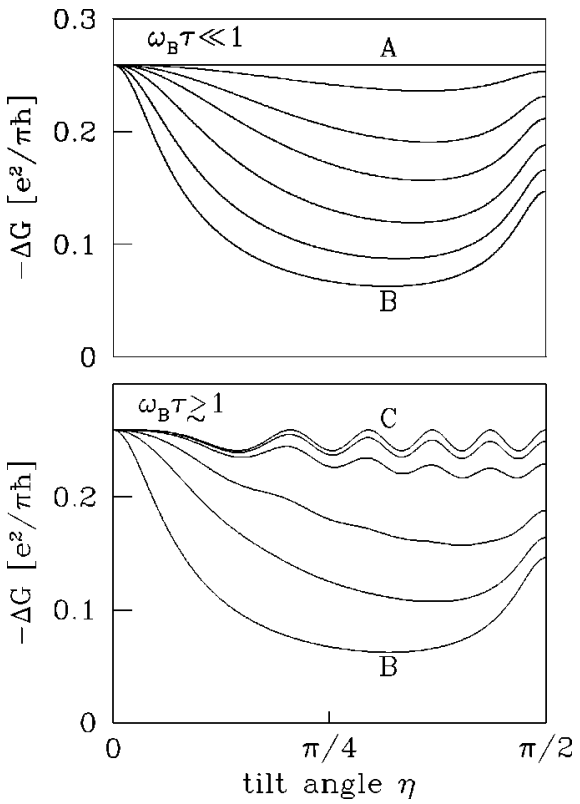

FIG. 3. Weak-localization correction $\Delta G$ of a ring in a spatially rotating magnetic field, as a function of the tilt angle $\eta$. Plotted is the result of Eq. (3.21) for $f=5, L=500 \ell, L_{\varphi}=125 \ell$. The upper panel is for $\omega_{\mathrm{B}} \tau \ll 1$. From top to bottom: $\omega_{\mathrm{B}} \tau=10^{-5}, 10^{-4}, 2$ $\times 10^{-4}, 3 \times 10^{-4}, 5 \times 10^{-4}, 10^{-3}, 10^{-2}$. At $\omega_{\mathrm{B}} \tau \simeq(f \ell / L)^{2}$, the weak-localization correction crosses over from the transient regime $A$ of Eq. (3.23) to the randomized regime $B$ of Eq. (3.30). The lower panel is for $\omega_{\mathrm{B}} \tau \gtrsim 1$. From bottom to top: $\omega_{\mathrm{B}} \tau=0.1,1,2,5,10$, 100. Here the weak-localization correction reaches the adiabatic regime $C$ of Eq. (3.22).

The transformed moments obey

$$
\begin{aligned}
\ell \frac{d \widetilde{N}_{\alpha \beta \gamma \delta}}{d x}= & \sum_{\alpha^{\prime}, \gamma^{\prime}}\left(T_{\alpha \alpha^{\prime}} \delta_{\gamma \gamma^{\prime}}+\delta_{\alpha \alpha^{\prime}} T_{\gamma \gamma^{\prime}}\right) \tilde{N}_{\alpha^{\prime} \beta \gamma^{\prime} \delta} \\
& +\sum_{\alpha^{\prime}, \gamma^{\prime}}\left(S_{\alpha \alpha^{\prime}} \delta_{\gamma \gamma^{\prime}}-\delta_{\alpha \alpha^{\prime}} S_{\gamma \gamma^{\prime}}\right) \widetilde{J}_{\alpha^{\prime} \beta \gamma^{\prime} \delta} \\
& -\left(1+\tau / \tau_{\varphi}\right) \widetilde{J}_{\alpha \beta \gamma \delta}, \\
\frac{d \widetilde{J}_{\alpha \beta \gamma \delta}}{d x}= & 2 \sum_{\alpha^{\prime}, \gamma^{\prime}}\left(S_{\alpha \alpha^{\prime}} \delta_{\gamma \gamma^{\prime}}-\delta_{\alpha \alpha^{\prime}} S_{\gamma \gamma^{\prime}}\right) \widetilde{N}_{\alpha^{\prime} \beta \gamma^{\prime} \delta} \\
& +\sum_{\alpha^{\prime}, \gamma^{\prime}}\left(T_{\alpha \alpha^{\prime}} \delta_{\gamma \gamma^{\prime}}+\delta_{\alpha \alpha^{\prime}} T_{\gamma \gamma^{\prime}}\right) \widetilde{J}_{\alpha \gamma^{\prime} \beta \gamma^{\prime} \delta} \\
& -\left(2 \tau / \tau_{\varphi}\right) \tilde{N}_{\alpha \beta \gamma \delta}+2 \tau \delta_{\alpha \beta} \delta_{\gamma \delta} \delta\left(x-x_{\mathrm{i}}\right),
\end{aligned}
$$

with the same $2 \times 2$ matrices $S$ and $T$ as in Sec. II. Because the transformation from $N$ to $\tilde{N}$ is unitary, the weaklocalization correction is still given by $\Delta G=$ $-\left(e^{2} D / \pi \hbar L\right) \Sigma_{\alpha, \beta} \widetilde{N}_{\alpha \beta \beta \alpha}\left(x_{\mathrm{i}}\right)$, as in Eq. (3.19).

We have solved Eq. (3.21) with periodic boundary conditions by numerically computing the eigenvalues and (right) eigenvectors of the $8 \times 8$ matrix of coefficients. The resulting $\Delta G$ is plotted in Fig. 3 as a function of the tilt angle $\eta$. In the adiabatic regime $\omega_{\mathrm{B}} \tau \gg f$ we find the conductance oscillations due to the Berry phase. These are given by ${ }^{6}$

$$
\Delta G=-\frac{e^{2}}{\pi \hbar} \frac{L_{\varphi}}{L} \frac{\sinh \left(L / L_{\varphi}\right)}{\cosh \left(L / L_{\varphi}\right)-\cos (2 \pi f \cos \eta)}
$$

analogously to the Aharonov-Bohm oscillations. ${ }^{4}$ (The length $L_{\varphi}=\sqrt{D \tau_{\varphi}}$ is the phase-coherence length.) In the randomized regime $(f \ell / L)^{2} \ll \omega_{\mathrm{B}} \tau \ll f$ there are no conductance oscillations. Instead we find a reduction of the weaklocalization correction, due to dephasing by spin scattering. In the transient regime $\omega_{\mathrm{B}} \tau \ll(f \ell / L)^{2}$ the effect of the field on the spin can be ignored, ${ }^{10}$ and the weak-localization correction remains at its zero-field value

$$
\Delta G=-\frac{e^{2}}{\pi \hbar} \frac{L_{\varphi}}{L} \operatorname{cotanh}\left(\frac{L}{2 L_{\varphi}}\right) .
$$

\section{Comparison with Loss, Schoeller, and Goldbart}

If we replace the Boltzmann operator $\mathcal{B}$ in Eq. (3.5) by the diffusion operator $-D \partial^{2} / \partial x^{2}$ and integrate over $\phi$ and $\phi_{i}$, we end up with the diffusion equation studied by LSG,

$$
\begin{gathered}
\left(\frac{\partial}{\partial t}-\mathcal{H}\right) \chi_{\alpha \beta \gamma \delta}\left(x, x_{\mathrm{i}} ; t\right)=\delta(t) \delta\left(x-x_{\mathrm{i}}\right) \delta_{\alpha \beta} \delta_{\gamma \delta} \\
\mathcal{H}=D \frac{\partial^{2}}{\partial x^{2}}+\frac{i g \mu_{\mathrm{B}}}{2 \hbar}\left[\mathbf{B}(x) \cdot \boldsymbol{\sigma}_{1}-\mathbf{B}(x) \cdot \boldsymbol{\sigma}_{2}\right], \\
\chi_{\alpha \beta \gamma \delta}\left(x, x_{\mathrm{i}} ; t\right)=\frac{1}{2 \pi} \int_{0}^{2 \pi} d \phi \int_{0}^{2 \pi} d \phi_{\mathrm{i}} \chi_{\alpha \beta \gamma \delta}\left(x, x_{\mathrm{i}} ; \phi, \phi_{\mathrm{i}} ; t\right) .
\end{gathered}
$$

Here $\boldsymbol{\sigma}_{1}$ and $\boldsymbol{\sigma}_{2}$ act, respectively, on the first and third indices of $\chi_{\alpha \beta \gamma \delta}$.

The difference between the diffusion equation (3.24) and the diffusion equation (3.15) is that Eq. (3.24) holds only if $\omega_{\mathrm{B}} \tau \ll 1$, while Eq. (3.15) holds for any value of $\omega_{\mathrm{B}} \tau$. LSG used Eq. (3.24) to argue that there exists an adiabatic region within the regime $\omega_{\mathrm{B}} \tau \ll 1$. In contrast, our analysis of Eq. (3.15) shows that adiabaticity is not possible if $\omega_{B} \tau \ll 1$. The argument of LSG is based on a mapping of the diffusion equation (3.24) onto the Schrödinger equation studied in Ref. 11. However, the mapping is not carried out explicitly. In this subsection we will solve Eq. (3.24) exactly using this mapping, to demonstrate that the adiabatic regime of LSG is in fact the randomized regime $B$. This misidentification perhaps occurred because both regimes are stationary with respect to the magnetic-field strength (cf. Fig. 2). However, Berry-phase oscillations of the conductance are only supported in the adiabatic regime $C$, not in the randomized regime $B$ (cf. Fig. 3).

We solve Eq. (3.24) for the weak-localization correction

$$
\Delta G=-\frac{e^{2} D}{\pi \hbar L} \sum_{\alpha, \beta}\left\langle x, \alpha, \beta\left|\left(\tau_{\varphi}^{-1}-\mathcal{H}\right)^{-1}\right| x, \beta, \alpha\right\rangle,
$$

where we introduced the basis of eigenstates $|x, \alpha, \beta\rangle$ (with $\alpha, \beta= \pm 1$ ) of the position operator $x$ and the spin operators $\sigma_{1 z}$ and $\sigma_{2 z}$. The operator $\mathcal{H}$ commutes with 


$$
J=\frac{L}{2 \pi i} \frac{\partial}{\partial x}+\frac{1}{2} f\left(\sigma_{1 z}+\sigma_{2 z}\right) .
$$

It is therefore convenient to use as a basis, instead of the eigenstates $|x, \alpha, \beta\rangle$, the eigenstates $|j, \alpha, \beta\rangle$ of $J, \sigma_{1 z}$, and $\sigma_{2 z}$. The eigenvalue $j$ of $J$ is an integer because of the periodic boundary conditions. The eigenfunctions are given by

$$
\begin{aligned}
& \left\langle x, \alpha^{\prime}, \beta^{\prime} \mid j, \alpha, \beta\right\rangle \\
& =\frac{1}{\sqrt{L}} \delta_{\alpha^{\prime} \alpha} \delta_{\beta^{\prime} \beta} \exp \left[\frac{2 \pi i x}{L}\left(j-\frac{1}{2} f \alpha-\frac{1}{2} f \beta\right)\right] .
\end{aligned}
$$

In the basis $\{|j, 1,1\rangle,|j, 1,-1\rangle,|j,-1,1\rangle,|j,-1,-1\rangle\}$ the operator $\mathcal{H}$ has matrix elements

$$
\begin{aligned}
&\left\langle j^{\prime}, \alpha^{\prime}, \beta^{\prime}|\mathcal{H}| j, \alpha, \beta\right\rangle=-D\left(\frac{2 \pi}{L}\right)^{2} \delta_{j^{\prime} j}\left(\begin{array}{ccccc}
(j-f)^{2} & 0 & 0 & 0 \\
0 & j^{2} & 0 & 0 \\
0 & 0 & j^{2} & 0 \\
0 & 0 & 0 & (j+f)^{2}
\end{array}\right) \\
&-i \omega_{\mathrm{B}} \delta_{j^{\prime} j}\left(\begin{array}{ccccc}
0 & \sin \eta & -\sin \eta & 0 \\
\sin \eta & -2 \cos \eta & 0 & -\sin \eta \\
-\sin \eta & 0 & 2 \cos \eta & \sin \eta \\
0 & -\sin \eta & \sin \eta & 0
\end{array}\right) .
\end{aligned}
$$

Substitution into Eq. (3.25) yields

$$
\begin{aligned}
\Delta G= & -\frac{e^{2} D}{\pi \hbar} \frac{1}{L^{2}} \sum_{\alpha, \beta} \sum_{j=-\infty}^{\infty}\left\langle j, \alpha, \beta\left|\left(\tau_{\varphi}^{-1}-\mathcal{H}\right)^{-1}\right| j, \beta, \alpha\right\rangle \\
= & -\frac{e^{2}}{\pi \hbar} \frac{1}{2 \pi^{2}} \sum_{j=-\infty}^{\infty}\left[\left(\gamma+j^{2}\right)^{2}\left(f^{2}+\gamma+j^{2}\right)\right. \\
& \left.+\kappa^{2}\left(3 f^{2}+4 \gamma+4 j^{2}+f^{2} \cos 2 \eta\right)\right] \\
& \times\left[\left(\gamma+j^{2}\right)^{2}\left(f^{4}+2 f^{2} \gamma+\gamma^{2}-2 f^{2} j^{2}+2 \gamma j^{2}+j^{4}\right)\right. \\
& +2 \kappa^{2}\left(f^{4}+3 f^{2} \gamma+2 \gamma^{2}-f^{2} j^{2}+4 \gamma j^{2}\right. \\
& \left.\left.+2 j^{4}+f^{2}\left(f^{2}+\gamma-3 j^{2}\right) \cos 2 \eta\right)\right]^{-1}
\end{aligned}
$$

We abbreviated $\kappa=2 \omega_{\mathrm{B}} \tau(L / 2 \pi \ell)^{2}$ and $\gamma=\left(L / 2 \pi L_{\varphi}\right)^{2}$. The sum over $j$ can be done analytically for $\kappa \gg 1$, with the result

$$
\begin{gathered}
\Delta G=-\frac{e^{2}}{\pi \hbar} \frac{1}{4 \pi Q}\left[\frac{4 a_{-}+4 \gamma+(3+\cos 2 \eta) f^{2}}{\sqrt{a_{-}} \tan \pi \sqrt{a_{-}}}\right. \\
\left.-\frac{4 a_{+}+4 \gamma+(3+\cos 2 \eta) f^{2}}{\sqrt{a_{+}} \tan \pi \sqrt{a_{+}}}\right], \\
Q=\left[f^{4}\left(9 \cos ^{2} 2 \eta-2 \cos 2 \eta-7\right)\right. \\
\left.-32 \gamma f^{2}(1+\cos 2 \eta)\right]^{1 / 2}, \\
a_{ \pm}=-\gamma+\frac{1}{4}(1+3 \cos 2 \eta) f^{2} \pm \frac{1}{4} Q .
\end{gathered}
$$

We have checked that our solution (3.29) of Eq. (3.24) coincides with the solution of Eq. (3.15) in the regime $\omega_{\mathrm{B}} \tau$ $\ll 1$. (The two sets of curves are indistinguishable on the scale of Fig. 3.) In particular, Eq. (3.30) coincides with the curves labeled B in Fig. 3, demonstrating that it represents the randomized regime-without Berry-phase oscillations.

Recently ${ }^{12}$ Loss, Schoeller, and Goldbart have reconsidered the condition for adiabaticity. We agree on the equations [our exact solution (3.29) is their starting point], but differ in the interpretations. They interpret our randomized regime $B$ as being the adiabatic regime and explain the absence of Berry-phase oscillations as being due to the effects of field-induced dephasing. We reserve the name "adiabatic' for regime $C$, because if the spin would follow the magnetic field adiabatically in regime $B$, it should not suffer dephasing.

\section{CONCLUSIONS}

In conclusion, we have computed the effect of a nonuniform magnetic field on the spin polarization (Sec. II) and weak-localization correction (Sec. III) in a disordered conductor. We have identified three regimes of magnetic-field strength: the transient regime $\omega_{\mathrm{B}} \tau \ll(f \ell / L)^{2}$, the randomized regime $(f \ell / L)^{2} \ll \omega_{\mathrm{B}} \tau \ll f$, and the adiabatic regime $\omega_{\mathrm{B}} \tau \gg f$. In the transient regime (labeled $A$ in Figs. 2 and 3), the effect of the magnetic field can be neglected. In the randomized regime (labeled $B$ ), the depolarization and the suppression of the weak-localization correction are maximal. In the adiabatic regime (labeled $C$ ), the polarization is restored and the weak-localization correction exhibits oscillations due to the Berry phase.

The criterion for adiabaticity is $\omega_{\mathrm{B}} t_{\mathrm{c}} \gg 1$, with $\omega_{\mathrm{B}}$ the spin-precession frequency and $t_{\mathrm{c}}$ a characteristic timescale of the orbital motion. We find $t_{\mathrm{c}}=\tau$, in agreement with Stern, ${ }^{3}$ but in contradiction with the result $t_{\mathrm{c}}=\tau(L / \ell)^{2}$ of Loss, Schoeller, and Goldbart. ${ }^{6}$ By solving exactly the diffusion equation for the Cooperon derived in Ref. 6, we have demonstrated unambiguously that the regime that in that paper was identified as the adiabatic regime is in fact the randomized regime $B$-without Berry-phase oscillations.

We have focused on transport properties, such as conductance and spin-resolved transmission. Thermodynamic properties, such as the persistent current, in a non-uniform magnetic field have been studied by Loss, Goldbart, and Balatsky $^{11,2}$ in connection with Berry-phase oscillations. These papers assumed ballistic systems. We believe that the adiabaticity criterion $\omega_{\mathrm{B}} \tau \gg 1$ for disordered systems should apply to thermodynamic properties as well as transport properties. This strong-field criterion presents a pessimistic outlook for the prospect of experiments on the Berry phase in disordered systems.

\section{ACKNOWLEDGMENTS}

We are indebted to L. P. Kouwenhoven for bringing this problem to our attention, and to P. W. Brouwer, D. Loss, and A. Stern for useful discussions. This research was supported by the "Nederlandse organisatie voor Wetenschappelijk Onderzoek" (NWO) and by the "Stichting voor Fundamenteel Onderzoek der Materie', (FOM). 
*Present address: Philips Research Laboratories, 5656 AA Eindhoven, The Netherlands.

${ }^{1}$ M. V. Berry, Proc. R. Soc. London, Ser. A 392, 45 (1984).

${ }^{2}$ D. Loss, P. Goldbart, and A. V. Balatsky, Phys. Rev. Lett. 65, 1655 (1990); in Granular Nanoelectronics, edited by D. K. Ferry, J. R. Barker, and C. Jacoboni (Plenum, New York, 1991).

${ }^{3}$ A. Stern, Phys. Rev. Lett. 68, 1022 (1992); in Quantum Coherence and Reality, edited by J. S. Anandan and J. L. Safko (World Scientific, Singapore, 1992).

${ }^{4}$ B. L. Altshuler, A. G. Aronov, and B. Z. Spivak, Pis'ma Zh. Eksp. Teor. Fiz. 33, 101 (1981) [JETP Lett. 33, 94 (1981)]; D. Yu. Sharvin and Yu. V. Sharvin ibid. 34, 285 (1981) [ 34, 272 (1981)]; A. G. Aronov and Yu. V. Sharvin, Rev. Mod. Phys. 59, 755 (1987).

${ }^{5}$ A. Stern, in Mesoscopic Electron Transport, edited by L. P. Kouwenhoven, G. Schön, and L. L. Sohn (Kluwer, Dordrecht, 1997).
${ }^{6}$ D. Loss, H. Schoeller, and P. M. Goldbart, Phys. Rev. B 48, 15218 (1993).

${ }^{7}$ L. P. Kouwenhoven (private communication). Experiments in a ballistic system were reported by A. F. Morpurgo, J. P. Heida, T. M. Klapwijk, B. J. van Wees, and G. Borghs, Phys. Rev. Lett. 80, 1050 (1998).

${ }^{8}$ Yu. Lyanda-Geller, I. L. Aleiner, and P. M. Goldbart, Phys. Rev. Lett. 81, 3215 (1998).

${ }^{9}$ S. Chakravarty and A. Schmid, Phys. Rep. 140, 193 (1986).

${ }^{10}$ Because we include only the effect of the magnetic field on the spin, we do not find the suppression of weak localization due to time-reversal-symmetry breaking of the orbital motion, nor do we find the Aharonov-Bohm oscillations due to the coupling of the magnetic field to the charge.

${ }^{11}$ D. Loss and P. M. Goldbart, Phys. Rev. B 45, 13544 (1992).

${ }^{12}$ Loss, Schoeller, and Goldbart, cond-mat/9805128 (unpublished). 